\title{
Numerical Simulation of Combined Mixing and Separating Flow in Channel filled with Porous Media
}

\author{
R. B. Khokhar ${ }^{1,2, a}$, Y. K. Chen ${ }^{2, b}, Y X^{2, c}$ and R. K. Calay ${ }^{3, d}$ \\ ${ }^{1}$ Department of BSRS,Mehran University of Engineering and Technology, \\ Jamshoro, Sindh, Pakistan, \\ ${ }^{2}$ School of Engineering and Technology, \\ University of Hertfordshire, UK, \\ ${ }^{3}$ Faculty of Engineering \&Technology, \\ University College Narvik 8505, Norway. \\ ar.khokhar@herts.ac.uk, by.k.Chen@herts.ac.uk, ${ }^{\mathrm{c} y}$.2.xu@herts.ac.uk, ${ }^{\mathrm{d}} \mathrm{rkc} @$ hin.no.
}

Keywords: mixing-separating geometry, flow bifurcation, porous media, finite element method, Newtonian fluid.

\begin{abstract}
Various flow bifurcations are investigated for two dimensional combined mixing and separating geometry. These consist of two reversed channel flows interacting through a gap in the common separating wall filled with porous media of Newtonian fluids and other with unidirectional fluid flows. The Steady solutions are obtained through an unsteady finite element approach that employs a Taylor-Galerkin/pressure-correction scheme. The influence of increasing inertia on flow rates are all studied. Close agreement is attained with numerical data in the porous channels for Newtonian fluids.
\end{abstract}

\section{Introduction}

It has been observed that from last many the flows of Newtonian and non-Newtonian fluids through porous medium in complex geometries have remained a very important research topic. Furthermore, the flow through a porous media of Newtonian and non-Newtonian fluids is an interesting subject matter of industrial importance and particularly in various engineering problems. Normally, industrial problems are much harder to tackle and present complex flow phenomena. Particularly, in the field of processing applications presents many challenges for researchers. Examples of these industrial and engineering applications are petroleum industrial applications such as enhanced oil recovery, crude oil extraction, electronic cooling, transpiration cooling, drying processes, thermal insulations, porous bearing, solar collectors, heat pipes, nuclear reactors, groundwater flow, chemical reactors, packed bed reactors, biomechanics, ceramic processing, chromatography, Food, Pharmaceutical, biotechnology, filtration process, geothermal engineering, insulation, and many others [ $2,3,25]$. In many processes of the complicating factor is the use of the fluids, which exhibits very complex rheological behaviour. The literature shows that several investigators [2, 3, 21and 25] have studied the characteristics of the hydrodynamics as well as the thermal behaviour of Newtonian flows through porous channels.

In current research study, the transient hydrodynamics characteristics of the flow of Newtonian fluid inside horizontal parallel-plate channels filled with porous medium have been investigated. A sophisticated numerical scheme is employed to explore wide parameter ranges of inertia and relative flow rates are of specific interest to this study. In this study, by employing the same geometry [1,7] and modified one of unidirectional fluid flows this study extends the limited information on the variety of Newtonian fluid flow behaviour in the mixing and separating geometry as well as in the other one in channel filled with porous materials. 


\section{Problem specification}

The detail of the particular mixing and separating flow problem considered are given schematically in figure-1(a). First consists of two inlet and two outlet flows in a planar channel that is divided into two different sections by the intersection of two thin plates, placed horizontally in the same central plane of the geometry and at a separation gap width of $\beta$. Other unidirectional geometry has one inlet from left bottom arm and outlet from right top arm of the channel. In this study wide gap geometry with a separation gap width of $\beta=3 \mathrm{~L}$ where $\mathrm{L}$ is characteristic length taken as the height of a single inlet channel arm. This facilitates an investigation of the impact of gap width on resultant flow structure. The thickness of the plate is taken as $\alpha=0.0254 \mathrm{~L}$. A sufficiently long length of channel of $23 \mathrm{~L}$ is selected to reproduce fully developed entry and exit flow. We have preferred the wide gap geometry because in medium gap geometry and narrow gap geometry it was observed that flow characteristics do not have dramatic change.

The flow domain is discretised into triangular elements that are generated by a uniform conformal mapping technique. The mesh design is such that the minimum size of element is in the neighbourhood of the separation or gap region, this being $0.003 \mathrm{~L}$. A finite element mesh on the domain is presented in figure-1(b), where the total number of elements, nodes, boundary nodes, vertex nodes and degrees of freedom are 1328, 2853, 392, 763, and 6469 respectively.

$\mathrm{u}=\mathrm{v}=0$

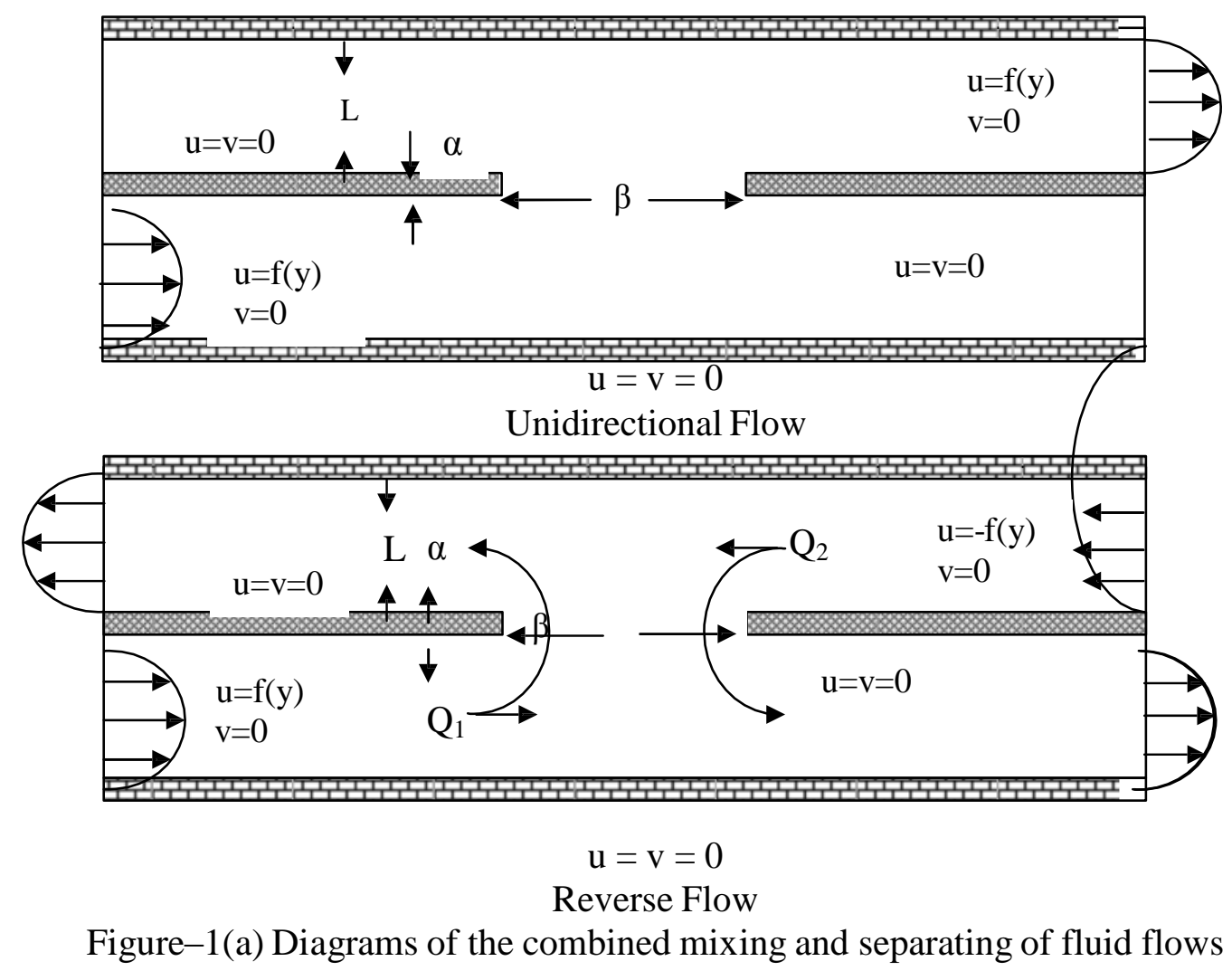




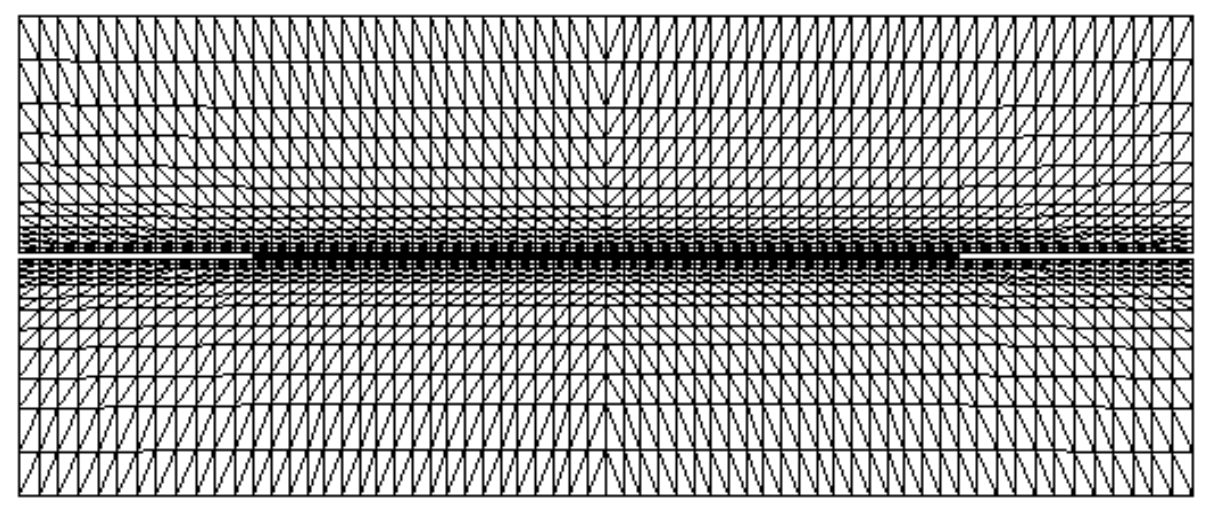

Figure-1(b): Finite element mesh used in the simulation.

The choice of appropriate time-step was made following the precursor study of [11], governed principally by an explicit time-stepping scheme Courant condition constraint that depends on a measure of the mesh spacing (taken as the minimum radius of encircle over the triangular elements). A semi-implicit method is implemented with three Jacobi mass-matrix iterations to capture an accurate solution and typical time steps involved is $\Delta t \leq 0.0001$ for Newtonian problems. The steady-state solutions are achieved at a time-step relative increment tolerance of $10^{-6}$ as in [11].

\section{Governing system of equation}

We consider a spatial bounded domain $\Omega \rightarrow \mathrm{R}^{2}$ with a piecewise smooth boundary $\Gamma$ and a temporal domain $[0, T]$ with $\mathrm{x}$ and $\mathrm{t}$ representing the associated spatial and time coordinates. In the absence of body forces, the corresponding equations for conservation of mass and Darcy-BrinkmanForchheimer momentum transport for an incompressible isothermal isotropic flow through homogeneous porous media may be stated as:

$$
\begin{array}{ll}
\nabla . \mathrm{u}=0, & \text { on } \Omega \mathrm{x}[0, \mathrm{~T}], \\
\frac{\rho}{\varepsilon} \frac{\partial \mathrm{u}}{\partial \mathrm{t}}=\nabla\left[2 \frac{\mu}{\varepsilon} \underline{\underline{\mathrm{D}}}\right]-\frac{\rho}{\varepsilon} \mathrm{u} . \nabla \mathrm{u}-\nabla \mathrm{p}-\frac{\mu}{\mathrm{K}} \mathrm{u} & \text { on } \Omega \mathrm{x}[0, \mathrm{~T}],
\end{array}
$$

or in non-dimensional form

$$
\frac{\partial \mathrm{u}}{\partial \mathrm{t}}=\frac{1}{\mathrm{Re}} \nabla^{2} \mathrm{u}-\mathrm{u} \cdot \nabla \mathrm{u}-\nabla \mathrm{p}-\frac{1}{\mathrm{Re} \mathrm{Da}} \mathrm{u}
$$

Where $u(x, t)$ is the fluid velocity vector field, $p(x, t)$ is the isotropic pressure (per unit density), $\rho$ and $\mu$ are the fluid density and viscosity respectively and $K$ is the intrinsic permeability of the porous medium. The $\mathrm{D}$ is the rate-of-deformation tensor, $\nabla$ is the gradient operator in the above equations (1), (2) and (3), the acceleration co-efficient tensor is assumed to be $\frac{1}{\varepsilon}$ and $\varepsilon$ is the porosity of the porous domain. Where $\quad D=\frac{1}{2}\left[\nabla u+(\nabla u)^{t}\right)$ and $t$ denotes matrix transposition. For Newtonian fluid $\mu$ is assumed as constant.

The flow is considered to be hydro-dynamically fully developed hence velocity does not depend on axial direction of the channel. As a result of the continuity equation, the flow is a unidirectional one and it is expressed in terms of axial velocity alone as function of transversal direction. Also, the pressure gradient is assumed to be constant. 
It is often convenient to cast the governing system of the equations in the form of non-dimensional form, using non-dimensional variables $\mathrm{x}^{*}, \mathrm{t}^{*}, \mathrm{u}^{*}, \mathrm{p}^{*}$, and $\mathrm{K}^{*}$. This may be achieved by selecting a suitable choice of characteristic scaling factors in the following manners:

$\mathrm{x}=\mathrm{Lx}^{*}, \mathrm{t}=\frac{\mathrm{L}}{\mathrm{U}} \mathrm{t}^{*}, \mathrm{u}=\mathrm{Uu}^{*}, \mathrm{p}=\frac{\rho \mathrm{U}^{2}}{\varepsilon} \mathrm{p}^{*}$ and $\mathrm{K}=\varepsilon \mathrm{L}^{2} \mathrm{~K}^{*}$, where $\mathrm{L}$ is a characteristic length taken as height of channel and $U$ is maximum inlet channel velocity. This leads to the following nondimensional groups of Reynolds number $\mathrm{Re}=\frac{\rho \mathrm{UL}}{\mu}$ and Darcy's number $\mathrm{D}_{\mathrm{a}}=\frac{\mathrm{K}}{\varepsilon \mathrm{L}^{2}}=\mathrm{k}^{*}$ by which can classify our study.

To complete the well posed problem specification it is necessary to prescribe the initial and boundary conditions. Simulations commence from quiescent initial conditions, or from two parallel but opposing flows, to obtain a steady-state solution for a fixed level of the inertial and elasticity parameters. Subsequently, to accelerate the computation times for higher parameter values, prior steady-state solutions are adopted as starting condition for higher parameter runs.

Initial conditions for the problem consist of specifying the value of $u$ at the initial time.

$$
\mathrm{u}(\mathrm{x}, 0)=\mathrm{u}_{0}(\mathrm{x}),(4 \mathrm{a})
$$

subject to

$$
\nabla \cdot \mathrm{u}_{0}=0 .
$$

In this case the pressure $\mathrm{p}$ is determined is up to an arbitrary constant.

The above equations (1-3) are supplemented by boundary conditions. Boundary conditions are taken as no slip on solid walls and inserted plates, and flow profiles are imposed at both entry flow sections to provide equal flow rates in both channel arms.

for porous media at both inlets an exact velocity profile is imposed as follows:

$$
\mathrm{u}(\mathrm{y})=\mathrm{V}_{\max }\left[1-\frac{\sinh \frac{\mathrm{y}-\mathrm{a}}{\sqrt{\mathrm{Da}}}+\sinh \frac{\mathrm{b}-\mathrm{y}}{\sqrt{\mathrm{Da}}}}{\sinh \frac{\mathrm{b}-\mathrm{a}}{\sqrt{\mathrm{Da}}}}\right], \text { on } \mathrm{x}=0 \text { (bottom left arm) and } \mathrm{x}=23 \mathrm{~L}
$$

(top right arm), where as Neauman boundary conditions are imposed at both exits. At both geometry exit sections, a traction free normal condition is enforced consistent with a fixed pressure. This approximation is found to be quite adequate through a careful check of the numerical flow fields derived in the fully developed flow regions, and in no way reduce the global accuracy of solutions.

\section{Numerical scheme and weak formulation}

To obtain steady solution we employ an unsteady Taylor-Galerkin/Pressure-correction technique. The Taylor-Galerkin/pressure-correction method was originally proposed at an early development stage by Townsend and Webster [8], and subsequently advanced by Hawken et al. [9] for Newtonian flows. More recently through the work of Carew et al. [10], this scheme has been extended in its range of application to cover highly elastic and complex flows.

Employing a Taylor-Galerkin/Pressure-correction technique algorithm for the governing system of equation (1-2) we obtain a semi-discrete system over a time step $\left[t_{n}, t_{n+1}\right]$. 
Stage 1a:

$\frac{2}{\Delta \mathrm{t}} \mathrm{u}^{\mathrm{n}+\overline{2}}-\mathrm{u}^{\mathrm{n}}=\left[\frac{1}{\operatorname{Re}} \nabla^{2} \cdot \mathrm{u}-(\mathrm{u} \cdot \nabla) \mathrm{u}-\nabla_{\mathrm{p}}+\frac{1}{\operatorname{Re} D \mathrm{Da}} \mathrm{u}\right]^{\mathrm{n}}$,

Stage 1b:

$\frac{1}{\Delta \mathrm{t}}\left(\mathrm{u}^{*}-\mathrm{u}^{\mathrm{n}}\right)=\left[\frac{1}{\operatorname{Re}} \nabla^{2} \cdot \mathrm{u}-\mathrm{u} \cdot \nabla \mathrm{v}-\frac{1}{\operatorname{ReDa}} \mathrm{u}\right]^{\mathrm{n}} \overline{-}-\nabla \mathrm{p}^{\mathrm{n}}(5 \mathrm{~b})$

Stage 2:

$$
\nabla^{2}\left(p^{n+1}-p^{n}\right)=\frac{1}{\Delta t} \nabla \cdot u^{*}(5 c)
$$

Stage 3:

$\frac{1}{\Delta \mathrm{t}}\left(\mathrm{u}^{\mathrm{n}+1}-\mathrm{u}^{*}\right)=-\nabla\left(\mathrm{p}^{\mathrm{n}+1}-\mathrm{p}^{*}\right) \cdot(5 \mathrm{~d})$

In above equations $\mathrm{n}$ represents the time step index. Velocity components at half step $\mathrm{n}+{ }^{1}$-are calculated in single step 1a from data at level $\mathrm{n}$ and in stage $1 \mathrm{~b}$ an intermediate non-solenoidal velocity $\mathrm{u}^{*}$ is calculated at the full time step, using solutions at the levels $\mathrm{n}$ and $\mathrm{n}+\frac{1}{\overline{2}}$ Solving a Poisson equation with the non-solenoidal velocity field $\mathrm{u}^{*}$, the pressure difference over full time step interval $[n, n+1]$ at stage 2 is evaluated and for second order accuracy in time the CrankNicolson choice $=0.5$ is adopted. Finally, at step three a solenoidal velocity at the end of the time step cycle from pressure difference field is captured. The inclusion of the half-step within the first fractional stage characterises the extension of the first to second-order projection method to TaylorGalerkin/pressure correction scheme.

Stage one and three are governed by augmented mass-matrices and solved by a Jacobi iterative method, that necessitates using only a small fixed number of mass iterations typically, three or so. At stage two, the Poisson equation matrix is symmetric and positive definite with a banded structure, for which it is appropriate to employ a direct Choleski method.

\section{Numerical Prediction and discussion}

\subsection{Newtonian flow in porous channels}

\subsubsection{The influence of inertia on flow structure}

In this study geometry of mixing and separating flows as shown in fig. 1(a) channel filled with porous material have been chosen. Here particular attention is paid to see any dramatic change in flow characteristics by opposing influences of flow inertia in channel filled with porous medium. Numerical simulations are given in figure 5.1.1for Newtonian fluid with increasing Reynolds number from $(01 \leq \operatorname{Re} \leq 5000)$ for equal flow rate.

Equal $(1,1)$ flow rate is analysed by presenting streamline patterns, which are not plotted at equal intervals in two flow regions, those of unidirectional and reversed flow. In our numerical program power law index is one, permeability is taken as 0.0001 and porosity is 1.0 . In the mixing region contours are plotted from the separation line to centrally located plate and in the unidirectional flow region from the channel wall to the separation to the separation line. Numerical simulations have been started from $\mathrm{Re}=01$ the flow is observed to respond to the presence of the gap and breaks up, with some flow unidirectional and most of the flow reversing and mixing in both upper and lower exit sections of the geometry. By adopting a continuation approach in the value of Reynolds number, solutions are obtained up to 1000 without any significant change in the flow structure. By Further increase in Rewe observed very weak activity behind the centrally positioned insert plates 
in the exit flow channel arms. By applying the same increase in value of Reynolds number we observed the activity of vortex recirculation at the same positions of the insert plates at $\mathrm{Re}=5000$ and the further recirculation of vortex have given rise to two eddies near the insert plates at same locations as was the situation in equal flow rate in non-porous medium case. The small eddies have maintained their position inclined with the insert plates and seems they become more stable (cf. 5.1.1 at $\mathrm{Re}=5000$ ) beyond this we are not aware about inertia effects. Here there is no any evidence of strong vortex development behaviour for low Reynolds number and it is the indication of very low opposing inertia effects on flow structure and it was expected as literature review reveals for Newtonian fluids.
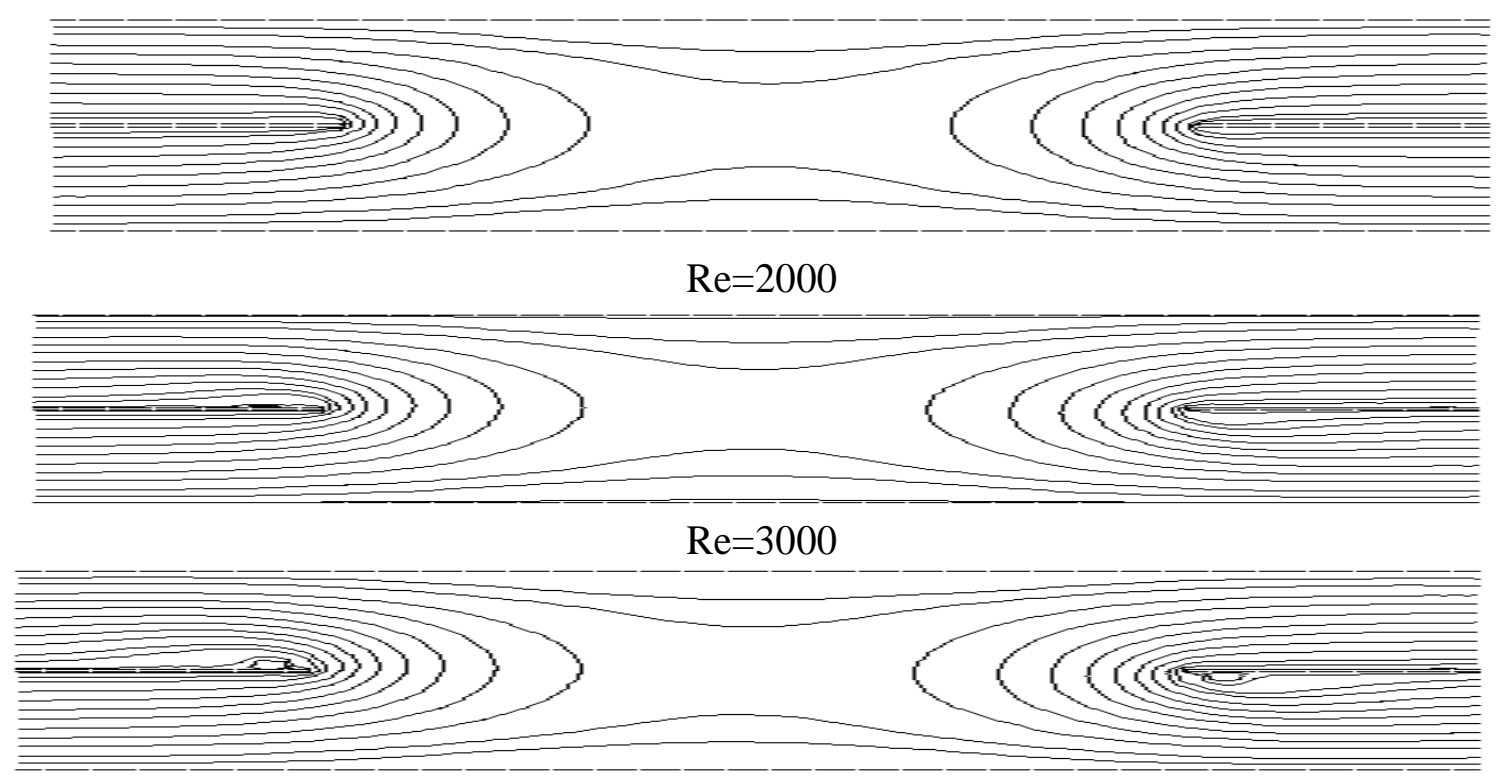

$\operatorname{Re}=5000$

Figure. 5.1.1 Streamline functions for Newtonian combined mixing and separating flow of equal flow rate $(1,1)$ in a both arms of channel filled with porous medium, increasing Re from top to bottom.

\subsection{Newtonian fluid flows in unidirectional geometry when channel filled with porous medium}

\subsubsection{The influence of inertia on flow structure}

Numerical predictions are given in fig. 5.2.1 for thegeometry of fig. 1 (a) of unidirectional fluid flows for $(01 \leq \operatorname{Re} \leq 6000)$. Simulations started with unit Reynolds number but we do not notice anything in either of the channel arms. By adopting the same continuation approach in the value of $\operatorname{Re}$ we found very late as well as very weak response of vortex development at $\mathrm{Re}=5000$ on the centrally positioned insert plates in the same side of top channel. Here both observed vortices are very weak but vortex on insert plate in the direction of flow channel is little bit stronger than the other located at insert plat of top arm of the same top arm of channel. By further increase in Re have not given us any significant effect but the vortex behind the insert plate in top arm in the direction of flow is little bit in more in size as compared with the remaining at an another adjacent insert plate. Results demonstrate the existence of very weak vortex behind the centrally positioned plate one in salient arm of top arm of channel and other behind the insert plate towards exit flow. Here there is no strong vortex development behaviour, very weak vortex is appears at very high value of Reynolds number. Here one thing which is surprisingly very different that vortices are on the same side of centrally positioned insert plates that is not the case in no porous medium, there vortices are on different sides of centrally positioned insert plates. 

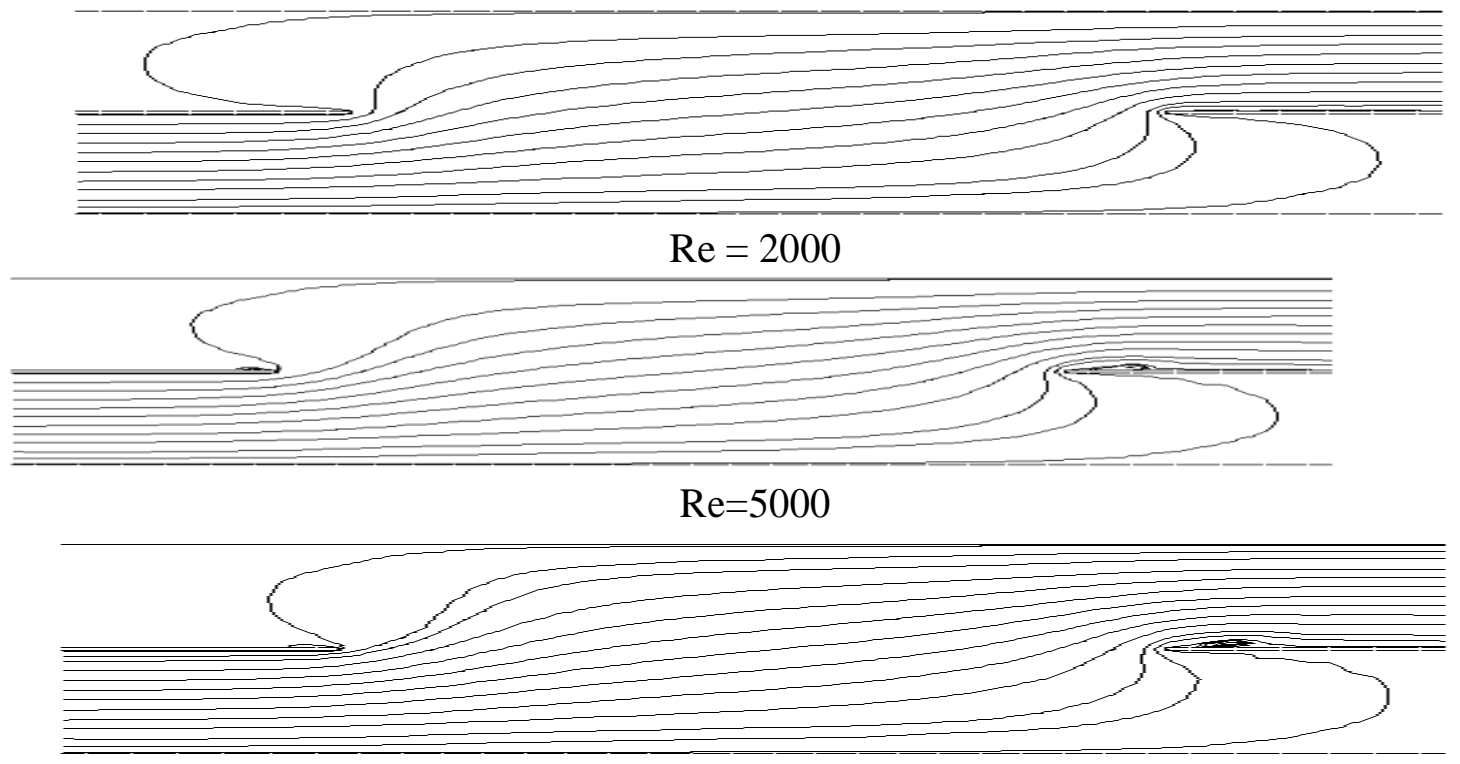

$\mathrm{Re}=6000$

Figure-5.2.1 Streamline functions of Newtonian fluid flow in channel filled with porous medium, increasing Re from top to bottom.

\section{Conclusion}

The semi-implicit time-stepping Taylor-Galerkin pressure correction primitive variable finite element algorithm has been found to be robust, stable and accurate in its predictions of steady fluid flows in channel filled with porous medium. From the results of two dimensional combined mixing and separating geometry and in unidirectional fluid flows algorithm gives adequate mesh convergence for the full compressible Navier-Stokes equation.

For monitoring the level of inertial effects the value of Reynolds number has been increased for equal flow rates in both geometries introduced in figure 1(a). It has been observed that increase in the value of Reynolds number has increased the intensity of the vortex development but activity is very slow when compared to channels filled without porous materials. Vortexes are very weak and small in size. At very high Reynolds numbers inertial effects were observed but process of meandering is not possible as was the case even with $\mathrm{Re}=200$ in case of channel filled with no porous medium in same geometries.

Therefore, it is predicted that inertial term has insignificant effects on the flow behaviour with power law index one and over the entire range of Darcy numbers which is in very good agreement with numerically results with [22]. It is observed that porous medium domains of low Darcy numbers have very small transient times for all ranges of microscopic inertial numbers and this implies that the effect of local inertia can be neglected because of they are having insignificant effects in porous domains. As the mass of fluid content in porous medium decreases its local inertia decreases.

\section{Acknowledgements}

The first author acknowledges the financial support under faculty development program from HEC and Mehran University of Engineering and Technology, Jamshoro, Sindh, Pakistan. 


\section{References}

[1] T. Cochrane, K. Walters, M. F. Webster, "On Newtonian and non- Newtonian flow in complex geometries”, Philos. Trans. R. Soc. London, A301, 163-181, 1981.

[2] S. Kakac, B. Kilkis, F. Arinic, "Convective Heat and Mass transfer in Porous media", Kulwer, Netherlands, 1991.

[3] D. A. Nield, A. Bejan, “Convection in Porous Media”, Springer-Verlag, New York, 1999.

[4] B.Alazmi, K. Vafai, "Analysis of Fluid flow and heat transfer interfacial conditions between a porous medium and fluid layer", Int. J. Heat and Mass transfer, 44, 1735-1749, 2001.

[5] A. Afonso, M. A. Alvis, R. J. Poole, P. J. Oliveira, and F. T. Pinho, "Viscoelastic lowReynolds-Number flows in Mixing-Separating Cells", Civil-Comp Press, Stirlingshire, Scoland, 1-12, 2008.

[6] K. Walters and M. F. Webster, "On dominating elasto-viscous response in some complex flows”, Philos. Trans. R. Soc. London, A308, 199-218, 1982.

[7] A. Baloch, P. Townsend, and M. F. Webster, "On the Simulation of Highly Elastic Complex Flows”, J. non-Newtonian Fluid Mech., 59(23), 111-128, 1995.

[8] A. Baloch, P. Townsend, and M. F. Webster, "Extensional Effects through Circular Contraction with Abrupt and Rounded re-Entrant Corners", JNNFM, 1994.

[9] P. Townsend, and M. F. Webster, "An Algorithm for the Three-Dimensional Transient Simulation of non-Newtonian Fluid Flows", In: theory and Applications, Proc. of Numeta Conf., Num. Meth. Eng., NUMETA87, 2, T 12/1-11 Nijhoff. 1987.

[10] D. M. Hawken, H. R. Tamaddon-Jahromi, P. Townsend, and M. F. Webster, "A TaylorGalerkin Based Algorithm for Viscous Incompressible Flow”, Int. J. Num. Meth. Fluids, 10, 327-351, 1990.

[11] E. O. A. Carew, P. Townsend and M. F. Webster, "A Taylor-Petro-Galerkin algorithm for viscoelastic flow", J. Non-Newtonian Fluid Mech., 50, 253-287, 1993.

[12] A. J. Chorin, "Numerical Solution of the Navier-Stokes Equations", Math. Comp., 22, 745$762,1968$.

[13] C. Cuvelier, A. Segal, and A. A. Van Steenhoven, "Finite Element Methods and Navier-Stokes Equations", D. Reidol, Dordrecht, Holland, 1986.

[14] J. Donea, “A Taylor-Galerkin Method for Convective Transport Problems”, Ins. J. Num. Meth. Eng., 20, 101-119, 1984.

[15] D. M. Hawken, H. R. Tamaddon-Jahromi, P. Townsend, and M. F. Webster, "A TaylorGalerkin Based Algorithm for Viscous Incompressible Flow", Int. J. Num. Meth. Fluids 10, 327-351, 1990.

[16] C. Johson, "Numerical Solution of Partial Differential Equations by the Finite Element Method" John Wiley and Sons, New York, 1990. 
[17] G. Strang, and G. F. Fix, "An Analysis of the Finite Element Method”, Englewood Cliffs N.J., Prence Hall, 1973.

[18] H. R. Tamaddon-Jahromi, D. P. Ding, M. F. Webster and P. Townsend, A Taylor Galerkin Finite Element Method for non-Newtonian Flows", Int. J. Num. Meth. Eng., 34, 741-757, 1992.

[19] J. Van Kan, "A Second-Order Accurate Pressure-Correction Scheme for Viscous Incompressible Flow”, SIAM J. Sci. Stat. Comput., 7, 870, 1986.

[20] P. Wapperom, M. F. Webster, "A second-order hybrid finite-element/volume method for viscoelastic flows", J. Non-Newtonian Fluid Mech., 79, 405, 1998.

[21] V. G. Ramanathan, "Estimating pressure drop in two-Phase flow through porous media",1-4, 2009.

[22] M. A. Al-Nimer, T. K. Aldos, "The effect of the macroscopic local inertial term on the nonNewtonian flow in channels filled with porous medium", International Journal of Heat and Mass Transfer, 47, 125-133, 2004.

[23] N. Fietier, "Numerical simulations of viscoelastic fluid flows by spectral element methods and time-dependent algorithm", PhD Thesis, Lausanne, EPFL, 2002.

[24] A. Baloch, "Numerical Simulation of complex flows of non-Newtonian fluids", PhD Thesis, University of Wales, Swansea, 1994.

[25] S. Kakac, B. Kilkis, F. Arinic, "Convective Heat and Mass transfer in Porous media", Kulwer, Netherlands, 1991.

[26] J. M. Marchal, M. J. Crochet, "A new mixed finite element for calculating viscoelastic flow", J. Non-Newtonian Fluid Mech., 26, 77-114, 1987.

[27] D. Rajagopalan, R.C, Armstrong, R. A. Brown, "Finite element method for calculation of steady viscoelastic flow using constitutive equation with a Newtonian viscosity", J. NonNewtonian Fluid Mech., 36, 77-159, 192, 1990.

[28] D. Rajagopalan, R.C, Armstrong, R. A. Brown, "Calculation of steady viscoelastic flow using a multimode Maxwell model: application of the explicit momentum equation (EMME) formulation”, J. Non-Newtonian Fluid Mech., 36, 135-157, 1990.

[29] Taha Sochi, "Modelling the flow of a Bautista-Manerofluid in porous media", Physics, fluid dynamics, 1-43, 2009.

[30] Anderson, J. D. Jr, “Computational Fluid dynamics: the basics with applications", McGrawHill Book Co Ltd, 1995.

[31] Jacob Bear, "Fluids in porous media", American Elsevier Publishing Company Inc., 1988. 